CAE Working Paper \#03-03

\title{
Aggregating Infinite Utility Streams with Inter-Generational
}

Equity: The Impossibility of Being Paretian

by

Kaushik Basu

\&

Tapan Mitra.

February 2003 


\title{
AGGREGATING INFINITE UTILITY STREAMS WITH INTER-GENERATIONAL EQUITY: THE IMPOSSIBILITY OF BEING PARETIAN*
}

\author{
Kaushik Basu ${ }^{1}$ and Tapan Mitra ${ }^{2}$
}

February 23, 2003

\begin{abstract}
It has been known that, in aggregating infinite utility streams, there does not exist any social welfare function, which satisfies the axioms of Pareto, inter-generational equity and continuity. We show that the impossibility result persists even without imposing the continuity axiom, and in frameworks allowing for more general domains of utilities than those used in the existing literature.

Journal of Economic Literature Classification Numbers: D00, D70, D90.

Keywords: Inter-generational equity, Pareto, social welfare functions.
\end{abstract}

${ }^{*}$ The authors are grateful to Jorgen Weibull for helpful suggestions on an earlier version of this paper. The current version has benefitted from comments by two referees of this journal.

${ }^{1}$ Department of Economics, Cornell University, Ithaca, NY 14853, Email: kb40@cornell.edu.

${ }^{2}$ Department of Economics, Cornell University, Ithaca, NY 14853, Email: tm19@cornell.edu. 


\section{Introduction}

The subject of inter-generational equity in the context of aggregating infinite utility streams has been of enduring interest to economists. Ramsey (1928) had maintained that discounting one generation's utility or income vis-a-vis another's to be "ethically indefensible", and something that "arises merely from the weakness of the imagination". Since it is generally agreed that any such process of aggregation should satisfy the Pareto principle, Ramsey's observation raises the question of whether one can consistently evaluate infinite utility streams while respecting intergenerational equity, and at least some form of the Pareto axiom.

In an important contribution to this literature, Svensson (1980) established the general possibility result (for a social welfare relation (SWR)) that one can find an ordering, that satisfies the axioms of Pareto and inter-generational equity. It is worth noting here that he obtains the (complete) ordering by non-constructive methods; specifically, he defines a partial order satisfying the two axioms, and then completes the order by appealing to Szpilrajn's lemma.

Svensson's paper builds on the earlier seminal contribution by Diamond (1965). In his paper, Diamond presents the celebrated theorem ${ }^{3}$ that there does not exist any social welfare function (SWF) (that is, a function that aggregates an infinite stream into a real number) satisfying three axioms: Pareto, intergenerational equity and continuity (in the sup metric).

Neither contribution addresses the following question: does there exist a social welfare function satisfying intergenerational equity, and the Pareto axiom? Since continuity of the SWF in Diamond's exercise is a technical axiom (in contrast to the other two axioms), we think that this is an issue worth investigating. 4 The principal task of this paper is to present the general

\footnotetext{
${ }^{3}$ Diamond attributes the result to Yaari.

${ }^{4}$ In this connection, we may note that the line of research, initiated by Koopmans (1960), and continued by Di-
} 
impossibility theorem that there is no SWF which satisfies the Pareto and equity axioms. In other words, we show (in Theorem 1) that any Paretian SWF is necessarily inequitable.

We can compare our result with Diamond's as follows. If we denote by $Y$ (a subset of the reals) the set of admissible utility levels of each generation, and by $X$ the set of infinite streams of these utility levels, an SWF is a function from $X$ to the reals. Our result implies that the continuity axiom is not needed for Diamond's impossibility theorem. Further, in our result, the utility space, $Y$, is unrestriced, while in Diamond's, it is taken to be the closed interval $[0,1]$. Thus, apart from the continuity issue, the context in which our result is established is broader.

Our approach to the impossibility result is different from Diamond's. His approach is to show that if there is a social welfare function on $X$ (when $Y=[0,1]$ ), satisfying equity and the Pareto principle, then it cannot be continuous in the sup-metric. The proof of our result, roughly speaking, involves showing that in trying to represent any social welfare relation respecting equity and the Pareto principle, one "runs out of real numbers". Thus, our approach has an affinity with the demonstration that lexicographic preferences do not have a real-valued representation.

Since the approaches are different, it is of some interest to enquire whether in Diamond's context (that is, with $Y=[0,1]$ ), one can dispense with the continuity axiom in his impossibility result, by following his own approach. We do this in Theorem 2. We show that the extent of continuity needed for Diamond's technique to work is already implied by the Pareto axiom in the $[0,1]$ utility space case, making a separate continuity axiom superfluous. ${ }^{5}$

amond (1965) and others, establishes that Paretian social welfare relations, continuous in suitably defined metrics, necessarily exhibit "impatience" in the sense that the current generation receives more favorable treatment than future generations. Burness (1973) explores the impatience implications of continuously differentiable Paretian social welfare functions.

${ }^{5}$ The underlying argument is that if an SWF were to exist, the Pareto axiom would imply that it would have some monotonicity properties, and monotone functions on $[0,1]$ can be discontinuous at most at countably many points. 
We establish (in Theorem 2) a stronger result than the one just described. We show that one does not need the full strength of the Pareto axiom; a weak version of it (and equity) suffices. This distinguishes it from Theorem 1, where the full strength of the Pareto axiom is needed.

\section{Paretian Social Welfare Functions are Inequitable}

Let $\mathbb{R}$ be the set of real numbers, $\mathbb{N}$ the set of positive integers, and $\mathbb{M}$ the set of non-negative integers. Suppose $Y \subset \mathbb{R}$ is the set of all possible utilities that any generation can achieve. Then $X=Y^{\mathbb{N}}$ is the set of all possible utility streams. If $\left\{x_{t}\right\} \in X$, then $\left\{x_{t}\right\}=\left(x_{1}, x_{2}, \ldots\right)$, where, for all $t \in \mathbb{N}, x_{t} \in Y$ represents the amount of utility that the $t^{t h}$ generation (that is, the generation of period $t$ ) earns. For all $y, z \in X$, we write $y \geq z$ if $y_{i} \geq z_{i}$, for all $i \in \mathbb{N}$; we write $y>z$ if $y \geq z$ and $y \neq z ;$ and we write $y>>z$, if $y_{i}>z_{i}$, for all $i \in \mathbb{N}$.

If $Y$ has only one element, then $X$ is a singleton, and the problem of ranking or evaluating infinite utility streams is trivial. Thus, without further mention, the set $Y$ will always be assumed to have at least two distinct elements.

A social welfare function (SWF) is a mapping $W: X \rightarrow \mathbb{R}$. Consider now the axioms that we may want the SWF to satisfy. The first axiom is the standard Pareto condition.

Pareto Axiom: For all $x, y \in X$, if $x>y$, then $W(x)>W(y)$.

The next axiom is the one that captures the notion of 'inter-generational equity'. We shall call it the 'anonymity axiom'. ${ }^{6}$ It is equivalent to the notion of 'finite equitableness' (Svensson, 1980) or 'finite anonymity' (Basu, 1994). ${ }^{7}$

\footnotetext{
${ }^{6}$ In informal discussions throughout the paper, the terms "equity" and "anonymity" are used interchangeably.

${ }^{7}$ The Anonymity Axiom figures prominently in the social choice theory literature, where it is stated as follows: the social ordering is invariant to the information regarding individual orderings as to who holds which preference
} 
Anonymity Axiom: For all $x, y \in X$, if there exist $i, j \in \mathbb{N}$ such that $x_{i}=y_{j}$ and $x_{j}=y_{i}$, and for $k \in \mathbb{N}-\{i, j\}, x_{k}=y_{k}$, then $W(x)=W(y)$.

The principal result of this paper can now be stated as follows.

Theorem 1 There does not exist any SWF satisfying the Pareto and Anonymity Axioms.

Proof. Assume, on the contrary, that there is a social welfare function, $W: X \longrightarrow \mathbb{R}$, which satisfies the Pareto and Anonymity Axioms. Since $Y \subset \mathbb{R}$ contains at least two distinct elements, there exist real numbers $y^{0}$ and $y^{1}$ in $Y$, with $y^{1}>y^{0}$. Without loss of generality, and to ease the writing, we may suppose that $y^{1}=1$ and $y^{0}=0$.

Let $Z$ denote the open interval $(0,1)$, and let $r_{1}, r_{2}, r_{3}, \ldots$ be an enumeration of the rational numbers in $Z$. Let $z$ be an arbitrary number in $Z$. We define the set: $E(z)=\left\{n \in \mathbb{N}: r_{n}<\right.$ $z$. Clearly, $E(z)$ is an infinite set. We now define a sequence $a(z)=\left(a(z)_{1}, a(z)_{2}, \ldots\right)$ as follows:

$$
a(z)_{n}= \begin{cases}1 & \text { if } n \in E(z) \\ 0 & \text { otherwise }\end{cases}
$$

Note that the sequence will have an infinite number of $1^{\prime} s$ and an infinite number of $0^{\prime} s$. Define the sequence $b(z)=\left(b(z)_{1}, b(z)_{2}, \ldots\right)$ as the same as the sequence $a(z)$ except that the first 0 appearing in the $a(z)$ sequence is replaced by a 1 . By the Pareto axiom, we have $W(b(z))>$ $W(a(z))$. We denote the closed interval $[W(a(z)), W(b(z))]$ by $I(z)$.

Now, let $p, q$ be arbitrary real numbers in $Z$, with $q>p$. Clearly, we must have $E(p) \subset E(q)$, for if $n \in E(p)$, then $r_{n}<p$, and since $p<q$, we must have $r_{n}<q$, so that $n \in E(q)$. Further,

ordering. Thus, interchanging individual preference profiles does not change the social preference profile. See May (1952) and Sen (1977). 
there are an infinite number of rational numbers in the interval $(p, q)$. Thus, comparing the sequence $a(p)$ with the sequence $a(q)$, we note that:

$$
\text { (i)if } n \in \mathbb{N} \text {, and } a(p)_{n}=1 \text {, then } a(q)_{n}=1 \text {, and }
$$

(ii)there are an infinite number of $n \in \mathbb{N}$, for which $a(p)_{n}=0$ and $a(q)_{n}=1$

We now proceed to compare the sequence $a(q)$ with the sequence $b(p)$. Let $m \in \mathbb{N}$ be the index for which the seqence $a(p)$ differs from the sequence $b(p)$; that is, $a(p)_{m}=0$, and $b(p)_{m}=1$. There are two cases to consider: (A) $a(q)_{m}=1,(\mathrm{~B}) a(q)_{m}=0 . \quad$ In case (A), we clearly have $a(q)>b(p)$, and so:

$$
W(a(q))>W(b(p))
$$

In case (B), we proceed as follows. Let $M$ be the smallest integer for which $a(p)_{M}=0$ while $a(q)_{M}=1$. By observation (2) above, such an $M$ exists. Also, clearly $M \neq m$, for $a(q)_{M}=1$ while $a(q)_{m}=0$. Since $b(p)$ differs from $a(p)$ in only the index $m$, we have $b(p)_{M}=0$. Now, define $b^{\prime}(p)$ as follows: $b^{\prime}(p)_{m}=0, b^{\prime}(p)_{M}=1, b^{\prime}(p)_{n}=b(p)_{n}$ for all $n \in \mathbb{N}$ such that $n \neq m, n \neq M$. Since $b(p)_{m}=1$, and $b(p)_{M}=0$, the Anonymity axiom implies that:

$$
W\left(b^{\prime}(p)\right)=W(b(p))
$$

Comparing $b^{\prime}(p)$ with $a(q)$, we note that $b^{\prime}(p)_{m}=0=a(q)_{m}, b^{\prime}(p)_{M}=1=a(q)_{M}$, and for all $n \in \mathbb{N}$ such that $n \neq m, n \neq M$, we have $b^{\prime}(p)_{n}=b(p)_{n}=a(p)_{n} \leq a(q)_{n}$ by observation (1). By 
observation (2), we must therefore have $a(q)>b^{\prime}(p)$, so that by the Pareto Axiom:

$$
W(a(q))>W\left(b^{\prime}(p)\right)
$$

Combining (4) and (5), we get:

$$
W(a(q))>W(b(p))
$$

Thus, in both cases $(\mathrm{A})$ and $(\mathrm{B})$, we have $W(a(q))>W(b(p))$. This means that the interval $I(q)=[W(a(q)), W(b(q))]$ is disjoint from the interval $[W(a(p)), W(b(p))]$, the latter interval lying entirely to the left of the former interval on the real line.

To summarize, we have shown that the intervals $I(z)$ associated with distinct values of $z \in$ $(0,1)$ are non-overlapping. But, this means that to each real number $z \in(0,1)$, we can associate a distinct rational number (in the interval $I(z)$ ), contradicting the countability of the set of rational numbers.

\section{Remarks:}

(i) The construction (used in the above proof) of an uncountable family of distinct nested sets $E(z)$, with each set containing an infinite number of positive integers, can be found in Sierpinski (1965, p. 82).

(ii) The general possibility theorem of Svensson (1980) on social welfare relations shows that there is no inherent conflict between the concepts of equity and the Pareto principle. Theorem 1 shows that a conflict arises when we try to obtain an evaluation of utility streams in terms of real numbers, while respecting these two properties.

(iii) The simplest example of our set-up is one where the utility space, $Y$, is $\{0,1\}$. One might 
interpret this as follows: there are precisely two states in which each generation might find itself, a good state and a bad state. The utility obtained by each generation is 1 in the good state, and 0 in the bad state. Theorem 1 tells us that even in this simple set-up, there is no SWF which respects Anonymity and the Pareto Axiom.

(iv) It is of interest to note that our general impossibility result does require the full strength of the Pareto Axiom. A weaker version of the Pareto axiom may be written as follows.

Weak Pareto Axiom: For all $x, y \in X$, if there exists $j \in \mathbb{N}$ such that $x_{j}>y_{j}$, and, for all $k \neq j$, $x_{k}=y_{k}$, then $W(x)>W(y)$. For all $x, y \in X$, if $x>>y$, then $W(x)>W(y)$.

It can be shown (see Basu and Mitra (2002, Theorem 3)) that when $Y \subset \mathbb{M}$ (the set of nonnegative integers), there is a social welfare function (on $X$ ) satisfying the Anonymity and Weak Pareto Axioms. ${ }^{8}$ In particular, when $Y=\{0,1\}$, there is an SWF on $X$, respecting Anonymity and the Weak Pareto Axiom.

\section{Generalizing the Diamond-Yaari Result}

Diamond (1965) had shown that (when the utility space $Y$ is $[0,1]$ ), there is no SWF satisfying Anonymity, Pareto, and a continuity axiom (in the sup metric on $X$ ). It follows, of course, from our Theorem 1 that the continuity axiom is redundant for his impossibility result.

The exercise in this section has two objectives. First, we show that the redundance of the continuity axiom for Diamond's impossibility result can be shown without any reference to our Theorem 1, but rather by following his own approach and exploiting more fully the particular

\footnotetext{
${ }^{8}$ Our Weak Pareto Axiom is stronger than the standard form of the Weak Pareto Axiom used in social choice theory, where typically it is stated as follows: if every individual in a society is better off, then society is better off. See, for example, Sen(1977). The possibility result just mentioned would, of course, hold with the standard form of the Weak Pareto Axiom in our context.
} 
structure of the utility space, when $Y$ is $[0,1]$. Second, we establish that (apart from the continuity axiom) the full power of the Pareto axiom is also not needed for his impossibility result; a weaker version of the Pareto axiom (which we call the dominance axiom) suffices for this purpose.

Dominance Axiom: For all $x, y \in X$, if there exists $j \in \mathbb{N}$ such that $x_{j}>y_{j}$, and, for all $k \neq j$, $x_{k}=y_{k}$, then $W(x)>W(y)$. For all $x, y \in X$, if $x>>y$, then $W(x) \geq W(y)$.

It is not as if we wish to recommend the use of such a weak form of the Pareto condition, but since we are going to prove an impossibility result, clearly it is better to use as weak an axiom as one can. Further, our proof indicates that it is precisely the Dominance axiom that is needed to obtain the impossibility result.

Theorem 2 Assume $Y=[0,1]$. There does not exist any $S W F$ satisfying the Dominance Axiom and the Anonymity Axiom.

Proof. To establish the theorem, assume $Y=[0,1]$ and that there exists a social welfare function, $W: X \rightarrow \mathbb{R}$, which satisfies the Dominance and Anonymity Axioms.

Denote the vector $(1,1,1, \ldots)$ in $X$ by $e$. Define the sequence $\bar{u}$ in $X$ as follows:

$$
\bar{u}=(1,1,0,1 / 2,1,0,1 / 4,2 / 4,3 / 4,1, \ldots)
$$

This sequence is best understood as sequence $u$, defined below, with the first term changed from 0 to 1 . For $s \in I \equiv(-0.5,0.5)$, define:

$$
\bar{x}(s)=0.5 \bar{u}+0.25(1+s) e
$$

Then $(1 / 8) e \leq \bar{x}(s) \leq(7 / 8) e$, and so $\bar{x}(s) \epsilon X$ for each $s \in I$. 
Define the function, $f: I \rightarrow \mathbb{R}$ by: $f(s)=W(\bar{x}(s))$. By the Dominance Axiom, $f$ is monotonic non-decreasing in $s$ on $I$. Thus $f$ has only a countable number of points of discontinuity in $I$. Let $a \epsilon I$ be a point of continuity of the function $f$.

Define the sequence $u$ in $X$ as follows:

$$
u=(0,1,0,1 / 2,1,0,1 / 4,2 / 4,3 / 4,1, \ldots)
$$

and then define:

$$
x(a)=0.5 u+0.25(1+a) e
$$

Clearly, $x(a) \epsilon X$, and $\bar{x}_{1}(a)>x_{1}(a)$, while $\bar{x}_{j}(a)=x_{j}(a)$ for each $j \epsilon \mathbb{N}$, with $j \neq 1$. By the Dominance Axiom, $W(\bar{x}(a))>W(x(a))$. We denote $[W(\bar{x}(a))-W(x(a))]$ by $\theta$; then $\theta>0$.

Denote $\max (0.5-a, 0.5+a)$ by $\triangle$; then, $\triangle>0$. Since $f$ is continuous at $a$, given the $\theta$ defined above, there exists $\delta \epsilon(0, \triangle)$, such that: $0<|s-a|<\delta$ implies $|f(s)-f(a)|<\theta$. Note that for $0<|s-a|<\delta$, we always have $s \in I$.

We define (following Diamond), for each $k \in \mathbb{N}$, a sequence $u^{k}$ by starting with the sequence $u$, interchanging the initial 0 with the $(k+1)$ st 1 appearing in $u$, and then interchanging the sequence $\left(1 / 2^{k}, 2 / 2^{k}, \ldots,\left(2^{k}-1\right) / 2^{k}, 0\right)$ with $\left(0,1 / 2^{k}, 2 / 2^{k}, \ldots,\left(2^{k}-1\right) / 2^{k}\right)$ so that:

$$
u^{k}=\left(1,1,0,1 / 2,1, \ldots, 0,0,1 / 2^{k}, 2 / 2^{k}, \ldots,\left(2^{k}-1\right) / 2^{k}, 0, \ldots\right)
$$

Now, for each $k \in \mathbb{N}$, we use $u^{k}$ to define $x^{k}(a)$ as follows:

$$
x^{k}(a)=0.5 u^{k}+0.25(1+a) e
$$


Clearly, $x^{k}(a) \in X$ for each $k \in \mathbb{N}$. Comparing the expressions for $x(a)$ and $x^{k}(a)$ in (10) and (12) respectively, and using the expressions for $u$ and $u^{k}$ in (9) and (11) respectively, we see that the Anonymity Axiom yields:

$$
W\left(x^{k}(a)\right)=W(x(a)) \quad \text { for all } k \in \mathbb{N}
$$

Choose $K \in \mathbb{N}$ with $K \geq 2$ such that $\left(1 / 2^{K-2}\right)<\delta$, and define $S=\left(a-\left(1 / 2^{K-2}\right)\right)$. We note that $0<(a-S)<\delta$, and so $S \in I$, and:

$$
W(\bar{x}(S))=f(S)>f(a)-\theta=W(\bar{x}(a))-\theta
$$

We now compare the welfare levels associated with $x^{K}(a)$ and $\bar{x}(S)$ as follows. Notice that $x^{K}(a)=0.5 u^{K}+0.25(1+a) e=0.5 \bar{u}+0.25(1+a) e-0.5\left(\bar{u}-u^{K}\right)=\bar{x}(a)-0.5\left(\bar{u}-u^{K}\right) \geq$ $\bar{x}(a)-0.5\left(1 / 2^{K}\right) e=0.5 \bar{u}+0.25(1+a) e-0.5\left(1 / 2^{K}\right) e=0.5 \bar{u}+0.25\left(1+a-\left(1 / 2^{K-1}\right)\right) e=$ $0.5 \bar{u}+0.25\left(1+a-\left(1 / 2^{K-2}\right)\right) e+0.25\left(1 / 2^{K-1}\right) e>>0.5 \bar{u}+0.25(1+S) e=\bar{x}(S)$. Thus, by the Dominance Axiom, we have:

$$
W\left(x^{K}(a)\right) \geq W(\bar{x}(S))
$$

Using (13), (14) and (15), we obtain: $W(\bar{x}(a))-\theta=W(x(a))=W\left(x^{K}(a)\right) \geq W(\bar{x}(S))>$ $W(\bar{x}(a))-\theta$, a contradiction, which establishes our result.

Remark: If we compare the Dominance Axiom with the Weak Pareto Axiom introduced in Section 2, we see that the last inequality in the statement of the former is a weak inequality, unlike in the statement of the latter. Hence, Weak Pareto is stronger than Dominance. It follows that when $Y=[0,1]$, there is no SWF satisfying the Weak Pareto and the Anonymity axioms. 


\section{References}

[1] Basu, K.: "Group Rationality, Utilitarianism and Escher's 'Waterfall' ", Games and Economic Behavior, 7 (1994), 1-9.

[2] Basu, K. and T. Mitra: "Aggregating Infinite Utility Streams with Inter-generational Equity", MIT Department of Economics Working Paper No. 02-19, 2002.

[3] Burness, H.S.: "Impatience and the Preference for Advancement in the Timing of Satisfactions", Journal of Economic Theory, 6 (1973), 495-507.

[4] Diamond, P.: "The Evaluation of Infinite Utility Streams", Econometrica, 33 (1965), 170177.

[5] Koopmans, T.C.: "Stationary Ordinal Utility and Impatience", Econometrica, 28 (1960), 287-309.

[6] May, K.O.: “A Set of Independent Necessary and Sufficient Conditions for Simple Majority Decision", Econometrica, 20 (1952), 680-684.

[7] Ramsey, F.P.: "A Mathematical Theory of Savings", Economic Journal, 38 (1928), 543-559.

[8] Sen, A.K.: "On Weights and Measures: Informational Constraints in Social Welfare Analysis", Econometrica, 45 (1977), 1539-72.

[9] Sierpinski, W.: Cardinal and Ordinal Numbers, Warsaw: Polish Scientific, 1965.

[10] Svensson, L.-G.: "Equity among Generations", Econometrica, 48 (1980), 1251-1256. 